\begin{tabular}{|c|c|c|c|c|c|}
\hline Intropica & ISSN 1794-161X & Vol. 13 & No.1 & $57-63$ & Santa Marta-Colombia, enero-junio de 2018 \\
\hline
\end{tabular}

\title{
DIAGNÓSTICO CLÍNICO DE MONOGENEOS EN ALEVINOS DE PISCICULTURA INTENSIVA EN ARAUCA
}

\author{
CLINICAL DIAGNOSIS OF MONOGENEA IN FINGERLING FISH FARM IN ARAUCA
}

\author{
Daniel Leonardo Cala Delgado (D), Norquis Caled Álvarez Rubio (D), Fabián Andrés Muñoz Rodríguez (D), \\ Carlos Alberto Blanco Torres $(D)$ Jefferson Yunis Aguinaga
}

\begin{abstract}
RESUMEN
Dentro de las enfermedades biológicas en la piscicultura, se encuentran las causadas por parásitos, principalmente por la clase monogenea, que provocan perdidas en la tasa de crecimiento y altas mortalidades, representando grandes pérdidas económicas en la acuicultura. Se reportó el diagnóstico clínico de parásitos monogeneos en peces producidos bajo sistemas intensivos, implementando tecnología biofloc (BFT) en dos municipios del departamento de Arauca. El primer caso se dio en la Asociación Piscícola El Vergel, municipio de Arauquita, en alevinos de tilapia roja (Oreochromis spp.). En el municipio de Arauca, en la Granja Experimental Picure (GEP), se presentó la segunda infestación parasitaria en alevinos de yamú (Brycon siebenthalae). En los dos casos, los parámetros de calidad físico-químicas de agua fueron evaluados con equipos y metodologías establecidas, la evaluación macro y microscópica de los peces fueron realizadas en el laboratorio de especies dulce acuícolas de la Universidad Cooperativa de Colombia sede Arauca. Los parámetros de calidad de agua se encontraron en los rangos ideales para la producción de peces, el único valor por debajo de lo recomendado fueron los sólidos decantables. Las lesiones macroscópicas encontradas fueron exoftalmia, ascitis, hemorragias, aletas y piel erosionada, en la evaluación microscópica, se observaron microorganismos parasitarios y por características morfológicas se logró establecer la presencia de monogeneos. Se instauro tratamiento con sal blanca en dosis de $3 \mathrm{~g} / \mathrm{L}$ de agua, se realizaron cambios del $50 \%$ del volumen total con agua desinfectada tres días antes usando formol a razón de $10 \mathrm{~mL} / \mathrm{L}$ de agua. La mortalidad fue del $95 \%$ de la población total de tilapia roja y el 100 \% de los alevinos de yamú. La desinfección del agua y alevinos antes de la siembra es lo más recomendable para evitar problemas ictiopatológicos.
\end{abstract}

PALABRAS CLAVE: sanidad; ictiopatología; biofloc; trematodos; desinfección

\begin{abstract}
Within the biological diseases in fish farming, there are those caused by parasites mainly by the monogeneous class causing great economic losses in aquaculture. The objective of this document is to report the clinical diagnosis of monogeneous parasites in fishes farmed under intensive systems, implementing biofloc technology (BFT) in two municipalities of the department of Arauca. The first case occurred in the Fish farming association of El Vergel, municipality of Arauquita, in red tilapia fingerlings (Oreochromis spp.). In the municipality of Arauca, at the Picure experimental farm (PEF), the second parasitic infestation occurs in yamu fingerlings (Brycon siebenthalae). In both cases, the parameters of physical and chemical quality of water were evaluated with equipment and methodologies established, the macro and microscopic evaluation of the fishes were carried out in the laboratory of freshwater species of the Universidad Cooperativa de Colombia based in Arauca. The parameters of water quality were found in the ideal ranges for fish production, the only value below the recommended was the settleable solids. The macroscopic lesions found were exophthalmos, ascites, hemorrhages, fins and eroded skin; in the microscopic evaluation were evident objects between gill lamellae that do not correspond to normal anatomical structures which agrees with parasitic microorganisms and by morphological characteristics it was possible to establish the presence of monogeneous in the studied fingerlings. Treatment with white salt at doses of $3 \mathrm{~g} / \mathrm{L}$ of water and changes of water pre-treated with formaldehyde at a rate of $10 \mathrm{ml} / \mathrm{L}$ of water was established but this did not work which caused the death of $95 \%$ of the total population of red tilapia and the $100 \%$ of the yamu fingerlings. The disinfection of water and fingerlings before sowing is the most recommended to avoid Ichthyopathological problems.
\end{abstract}

KEY WORDS: health; ichthyopathology; biofloc; parasitology; disinfection

Dirección de los autores:

Grupo de investigación Los Araucos, Facultad de Medicina Veterinaria Zootecnia, Universidad Cooperativa de Colombia, Sede Arauca. Kilómetro 1 vía Caño Limón, granja El Picure, Arauca, Colombia; e-mail: daniel.cala@campusucc.edu.co (D.L.C.D.); e-mail: fabian.munozro@campusucc. edu.co (F.A.M.R.). Facultad de Medicina Veterinaria Zootecnia. Universidad Cooperativa de Colombia, Sede Arauca. Kilómetro 1 vía Caño Limón, granja El Picure, Arauca, Colombia; e-mail: caled95@hotmail.com (N.C.A.R.); e-mail: kbttorres@yahoo.com.co (C.A.B.T.). Facultad de Ciencias Agrarias y Veterinarias. Universidad Estadual Paulista, Jaboticabal, San Pablo, Brasil. Vía de Acceso Prof. Paulo Donato Castellane s/n 14884-900, Jaboticabal, San Pablo, Brasil; e-mail: jefyunis@gmail.com (J.Y.A.). 
La producción acuícola generó en el 2014 valores superiores a 73,8 millones de toneladas de organismos acuáticos, representados en 160200 millones de USD, de los cuales la piscicultura aporta tres cuartas partes del volumen total de la acuicultura (FAO, 2016). Suramérica se conoce por su alta producción y exportación de organismos acuáticos, producto de la pesca y la acuicultura, pero no ha logrado alcanzar su pleno potencial en la piscicultura, donde se implementan principalmente sistemas extensivos y semi-intensivo (Valladão et al., 2016). La implementación de sistemas tradicionales de producción piscícola tiende a reducirse, debido al alto costo de alimento, uso ineficiente del agua, contaminación del recurso hídrico y otros inconvenientes ambientales (Wilfart et al., 2013). La tecnología biofloc (BFT) es un sistema intensivo para producción de organismos acuáticos que se basa en el recambio limitado de agua, el fundamento es el aprovechamiento de residuos de alimento, compuestos orgánicos e inorgánicos tóxicos, por medio de microorganismos presentes en el agua, dando condiciones propicias para bacterias quimiotótrofas, autótrofas y heterótrofas, que reducen la saturación de nutrientes para su reutilización (Avnimelech, 2009).

En los sistemas intensivos se reporta disminución de microorganismos oportunistas, siempre y cuando se realice una debida desinfección inicial de alevinos, unidades de producción y se controlen parámetros de calidad de agua (Monroy-Dosta et al., 2013). Asimismo, la desinfección del agua antes de la siembra es de vital importancia, Esto evita la introducción y propagación de enfermedades infecciosas en las granjas piscícolas (Kasai et al., 2002). Dentro de las enfermedades de origen biológico, se encuentran las causadas por parásitos, estos se pueden clasificar según la relación con el huésped, los que se localizan al interior del mismo se denominan endoparásitos y ectoparásitos cuando viven en la superficie del hospedante (Balbuena, 2011). Entre los parásitos externos más comunes de los peces se encuentran los de la clase Monogenea (Franceschini et al., 2013). Platelmintos pequeños que causan grandes pérdidas económicas en la acuicultura (Da Costa et al., 2017) cuyas cargas parasitarias altas causan lesiones epiteliales severas y alteraciones hematológicas.

(Tavares-Dias et al., 2008; Araújo et al., 2009b) sirviendo como puerta de entrada para infecciones secundarias por microorganismos oportunistas, lo que se representa en altas mortalidades (Huang et al., 2013), necesitando los tratamientos terapéuticos para minimizar las pérdidas 58 económicas causadas por infestaciones parasitarias
(Valladão et al., 2015). Los productos usados para contrarrestar los parásitos monogeneos son peróxido de hidrogeno (Bowker et al., 2012; Hirazawa et al., 2016), permanganato de potasio (Umeda et al., 2006), soluciones de formalina (Pahor-Filho et al., 2012) y praziquantel (Sitjà-Bobadilla et al., 2006; Forwood et al., 2013), para realizar esos tratamientos lo más importante es el diagnóstico definitivo del causante de la enfermedad (Raja y Jithendran, 2015).

Los monogeneos están distribuidos por todo Suramérica y afectan la gran mayoría de peces en los diferentes sistemas productivos (Valladão et al., 2016). El objetivo del trabajo es reportar el diagnóstico clínico de monogeneos branquiales en alevinos de tilapia roja (Oreochromis spp.) y yamú (Brycon siebenthalae) producidos bajo sistema intensivo (BFT) (figura 1), en los municipios de Arauca y Arauquita, del departamento de Arauca.

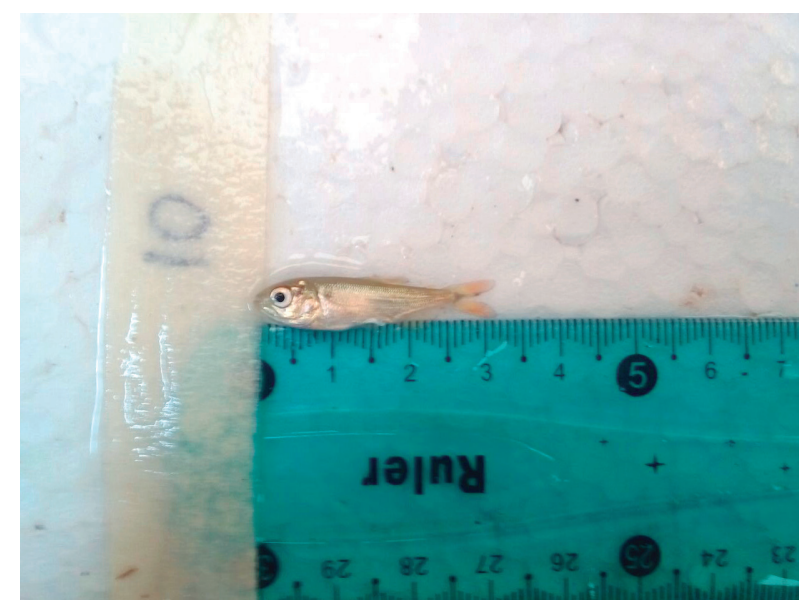

Figura 1. Fotografía de alevino de Yamú (Brycon siebenthalae), aparentemente sano.

El primer diagnóstico se realizó en la Asociación Piscícola El Vergel (Asovergel), ubicada en el municipio de Arauquita, vereda la Arenosa, donde se produce tilapia roja implementando el sistema BFT, con recambios de agua limitados, esta estación cuenta con 19 tanques de geomembrana en polietileno de alta densidad (HDPE), de 14 metros de diámetro. Esta unidad de producción tiene capacidad aproximada para producir entre 4 y 5 toneladas mensuales, de tilápia roja. El tanque de recepción de alevinos es de 7 metros de diámetro, donde se reciben 40000 alevinos por mes. Diariamente se evalúan los parámetros de calidad de agua, temperatura (T), oxígeno disuelto en concentración (ODC) y saturación (ODS), los cuales se miden con sonda YSI EcoSense ${ }^{\circledR}$ DO200A, pH, total de 
solidos disueltos (TDS), conductividad eléctrica (CE) con ayuda de equipo Hanna ${ }^{\circledR}$ modelo 991300, una vez por semana se evalúan los parámetros químicos, amonio $\left(\mathrm{NH}_{4}\right)$, nitrito $\left(\mathrm{NO}_{3}\right)$, nitrato $\left(\mathrm{NO}_{3}\right)$, alcalinidad (A), Ortofosfatos (O), con metodologías establecidas para cada parámetro y finalmente por medio de cono Imhoff se mide el volumen de solidos decantables (SD). Los productores reportaron $30 \%$ de mortalidad ocho días después del ingreso de 40000 alevinos de tilapia roja, adquiridos en un laboratorio certificado para la reproducción de peces, indicaron que no fue realizada la debida desinfección de los nuevos peces. Siguiendo lo recomendado por Balbuena (2011) en el Manual básico de sanidad piscícola, para la obtención y envió de muestras al laboratorio, en total cien alevinos vivos que manifestaban la enfermedad fueron transportados al laboratorio de especies dulce acuícolas de la Facultad de Medicina Veterinaria y Zootecnia (FMVZ), perteneciente a la Universidad Cooperativa de Colombia (UCC), sede Arauca. Los animales ingresaron a la unidad de cuarentena, en un tanque de concreto con capacidad de 1000 litros y aireados con motor tipo blower de 0,5 HP para mantener el oxígeno disuelto en niveles adecuados para conservar con vida los peces. Posterior a la aclimatación fue observado el comportamiento. Cinco peces vivos fueron retirados, en cajas Petri que contenían agua para el examen macroscópico y la valoración de lesiones, hisopados de piel y muestras branquiales de peces muertos fueron observados directamente sobre portaobjetos a $4 \mathrm{x}$ en microscopio de luz.

El segundo caso se presentó en la Granja Experimental el Picure (GEP) de la UCC, ubicada en el kilómetro 1 vía caño Limón del Municipio de Arauca. En los tanques de concreto para experimentación se adelantaba un estudio piloto de levante de peces nativos usando BFT, 600 alevinos de yamú, con peso $(0,35 \pm 0,047 \mathrm{~g})$ y talla $(3,2 \pm 0,052 \mathrm{~cm})$ (figura 2).

Transcurrido cinco días posteriores al inicio del experimento se reportaron mortalidades. Se procedió a evaluar los parámetros de calidad de agua, OD y T con sonda YSI ${ }^{\circledR}$ DO200 y pH con tester Hanna ${ }^{\circledR}$ 98128, Se cuantificó NH4 con tiras reactivas y los SD con cono Imhoff. Se inspeccionó semiológicamente mediante observación directa de alevinos vivos al microscopio (4x), evaluación comportamental y sobrevivencia. Los parámetros de calidad de agua en los dos casos se encontraron dentro de los rangos ideales para la producción de tilapia roja y yamú, implementando tecnología biofloc, el único parámetro que se encontró disminuido fue los sólidos decantables SD en tilapia roja (tabla 1).

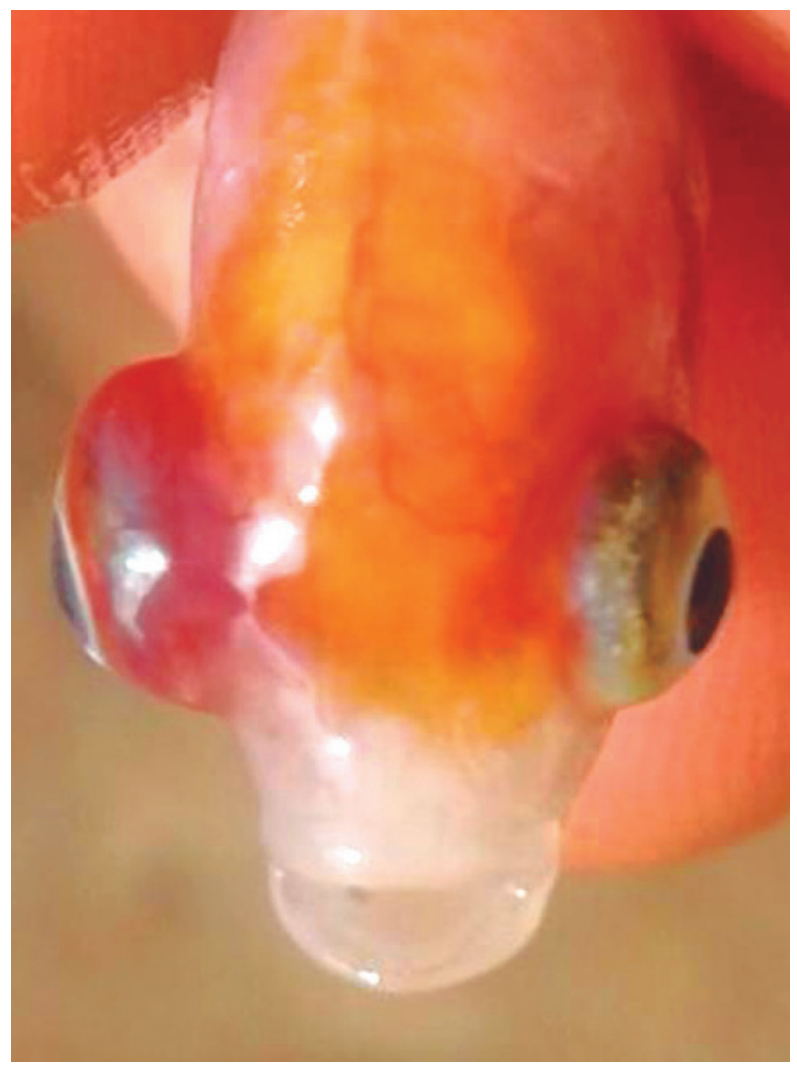

Figura 2. Tilapia roja (Oreochromis spp.) presentando exoftalmia bilateral marcada.

En la evaluación del comportamiento se observó que los animales perdían el equilibrio alejándose del cardumen, frecuencia opercular aumentada, inapetencia, nado errático y se frotaban contra las paredes de los tanques. La inspección semiológica evidenció hemorragias generalizadas, perdida de continuidad en aletas caudales, palidez branquial, mal olor, acumulación de líquido intraperitoneal (ascitis) y exoftalmia. Los dos últimos sugieren concomitancia con otras patologías del orden bacteriano o viral y se presentó solo en los alevinos de tilapia.

La evaluación microscópica de las branquias de peces afectados, confirmó coloración pálida, indicando anemia, branquitis necrótica multifocal y se observan objetos entre las lamelas branquiales anormales (figura 3), que por características morfológicas se identificaron como parásitos de la clase Monogenea, los parásitos observados en las branquias de tilapia y yamú, eran de forma ovalada tenían longitud aproximada de 365 y $68 \mu \mathrm{m}$. de ancho, estos presentaban dos pares de ojos ubicados en la región antero-dorsal y un órgano de fijación en la parte posterior con forma de disco dividido en ventosas compatible con un órgano denominado 
opistohaptor encontrado en el género Dactylogyrus sp. de la clase Monogenea. La evaluación microscópica de los alevinos y el hisopado de piel, sirvieron para observar que además de estar presentes en las branquias, también se encontraban parásitos distribuidos en aletas caudales y dorsales, con presencia en globos oculares.

Tabla 1. Resultados de parámetros físico-químicos del agua obtenidos de dos piscícolas intensivas de Arauca, durante el estudio de casos clínicos.

\begin{tabular}{lrrr}
\hline \multicolumn{4}{c}{ Parámetros de calidad de agua para BFT } \\
\hline \multicolumn{1}{c}{ Parámetros } & $\begin{array}{c}\text { Rango ideal } \\
\text { (Boyd y Massaut, 1999) }\end{array}$ & Asovergel & \multicolumn{1}{c}{ GEP } \\
\hline Oxígeno disuelto (ppm) & $>3$ & $4,5 \pm 0,3$ & $5 \pm 0,5$ \\
Oxígeno disuelto (\%) & $50-70$ & $58,1 \pm 9,3$ & $70 \pm 10$ \\
Temperatura $\left({ }^{\circ} \mathrm{C}\right)$ & $24-30$ & $28,5 \pm 1,9$ & $27 \pm 0,2$ \\
pH & $6,5-8$ & $7,3 \pm 0,3$ & $6,5 \pm 0,3$ \\
TDS (ppm) & $<1000$ & $991 \pm 123$ & - \\
CE ( $\mu$ S cm-1) & $<1000$ & $821 \pm 97$ & - \\
Amonio (ppm) & $<1$ & $0,25 \pm 0$ & 0 \\
Nitrito (ppm) & $<1$ & $0,1 \pm 0$ & - \\
Nitrato (ppm) & $10-200$ & $10 \pm 0,5$ & - \\
Ortofosfatos (mg/L) & $1-10$ & $0,25 \pm 0,1$ & - \\
Alcalinidad (ppm) & $>120$ & $123 \pm 13$ & - \\
SD (ppm) & $25-50$ & $5 \pm 0,5$ & $6 \pm 1,2$ \\
\hline
\end{tabular}

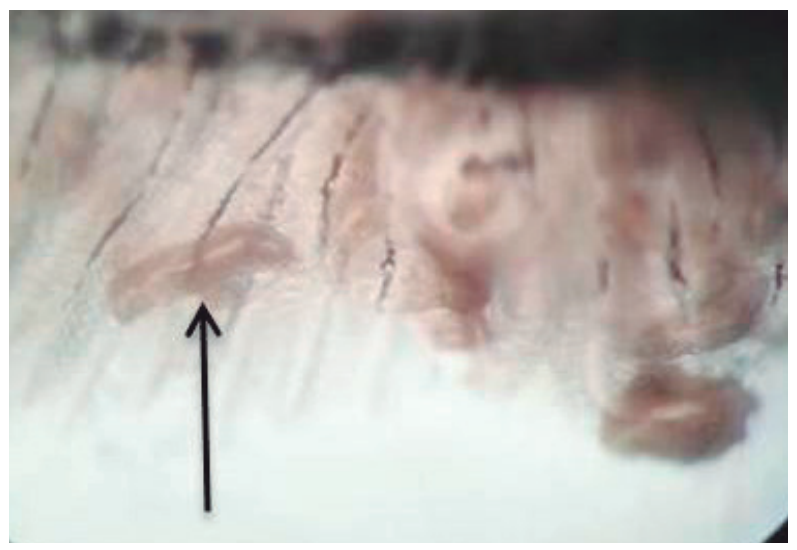

Figura 3. Parásito en las lamelas branquiales de yamú 60
A pesar del tratamiento instaurado con baños de sal blanca en dosis de $3 \mathrm{~g} / \mathrm{L}$ de agua durante cincuenta minutos por tres días seguidos y el uso de agua desinfectada en reservorio externo tres días previos al recambio con formol en dosis de $10 \mathrm{ml} / \mathrm{L}$, la mortalidad para el caso del sistema productivo de tilapia fue de $95 \%$, quedando solo 2000 alevinos de los 40000 sembrados, en el sistema intensivo experimental con yamú la mortalidad fue del $100 \%$.

Los parámetros de calidad de agua se encontraron dentro de lo recomendado para la producción de especies acuícolas, el único parámetro con valor disminuido fueron los SD, esto sucede cuando se inicia el proceso BFT de cero y se clasifica como floc inmaduro 
(Avnimelech, 2009). Una desventaja del floc inmaduro es que no desplaza comunidades de microorganismos oportunistas patógenos, como si lo hace el floc maduro (Crab et al., 2012). En los dos estudios de casos, se inició la etapa de alevinaje con SD en valores inferiores a lo recomendado, lo que beneficio el aumento de la carga parasitara, debido a su ciclo de vida monoxeno, con la desventaja que los parásitos monogeneos se reproducen con gran rapidez (Takemoto et al., 2004), esto aunado a los cambios limitados de agua que empeoraron la infestación parasitaria.

Las lesiones macroscópicas, concuerdan con los daños tisulares reportados por Araújo et al (2009b), hemorragias petequiales, perdida de continuidad en la piel y caída de las escamas también fueron indicadas como lesiones causadas por parásitos monogeneos en Gasterosteus aculeatus leiurus (Thoney y Hargis, 1991) Sin embargo, la exoftalmia, ascitis y olor putrefacto son consecuencia de infecciones secundarias por patógenos oportunistas, principalmente bacterias (Huang et al., 2013).

Los cambios hematológicos reportados por TavaresDias et al (2008), no se pudieron evidenciar en los dos casos del presente reporte, por dificultades en la colecta de sangre, debido al tamaño de los alevinos, pero la palidez de la estructura branquial, tanto para tilapia roja como para yamú, indican procesos anémicos, por las hemorragias generalizadas. Las pérdidas de continuidad tisular de las lamelas branquiales son causadas por los ganchos usados por el parasito para sujetarse al hospedador (Mendoza-Franco et al., 2010). Los parásitos distribuidos por el cuerpo evidenciaban la gran carga parasitaria, de la misma forma que en alevinos de Arapaima gigas, donde existió relación positiva entre el número de monogeneos en branquias y la longitud total del pez (Araújo et al., 2009a).

Los tratamientos comúnmente recomendados no surtieron efecto en estos casos, la sal en dosis de $3 \mathrm{~g} / \mathrm{l}$ por tres días no eliminaron la presencia del parásito, es por eso que en la búsqueda de tratamientos alternativos se consideran los aceites esenciales de plantas como la copaifera usados inmersión durante 10 minutos en Piaractus mesopotamicus con dosis de $50 \mathrm{mg} / \mathrm{l}$ de agua, reduciendo un $45 \%$ de la parasitosis (da Costa et al., 2017). La ineficiencia del tratamiento instaurado, provoco mortalidades altas, es común que el $100 \%$ de la población de peces parasitados en estado de confinamiento muera (Sarmiento y Rodríguez., 2013). Para evitar las pérdidas económicas en la piscicultura producida por parasitosis lo más recomendable es realizar desinfección del agua de recepción, alevinos y limpieza de equipos e instalaciones (Kasai et al., 2000; 2002)

La presencia de monogeneos en alevinos de tilapia roja y yamú en sistema intensivo fue altamente patógeno, desencadenando mortalidad alta que llevo a la pérdida total del cultivo, las herramientas de laboratorio son de vital importancia para el diagnóstico clínico, la dificultad de determinar el género del parásito disminuye la posibilidad de realizar tratamientos adecuados y el tiempo de exposición de los organismos a los monogeneos pueden permitir ingreso de otros patógenos como bacterias que produzcan lesiones como la ascitis. A pesar del auge que ofrecen los sistemas de biofloc en incremento de biomasa proveniente de la producción acuícola intensiva, esta nota científica desarrolla una importante descripción de los efectos negativos que puede tener el uso de un floc inmaduro, que se traduce en un incremento de la carga bacteriana que en cautiverio conducen a altas tasas de mortalidad.

\section{AGRADECIMIENTOS}

Los autores agradecen a la Asociación Piscícola el Vergel y cada uno de sus integrantes, por la información entregada en lo relacionado a los registros de calidad de agua y permitirnos realizar el diagnostico. De igual manera a la Empresa R \& R Soluciones S.A.S, por prestar equipos de medición de calidad de agua, facilitar el transporte y personal. A la Decanatura de la Facultad de Medicina Veterinaria y Zootecnia de la Universidad Cooperativa de Colombia sede Arauca, por permitir el uso de las instalaciones, equipos y reactivos.

\section{REFERENCIAS}

Araújo, C.S.O., Gomes, A.L., Tavares-Dias, M., Andrade, S.M.S., Belem-Costa, A., Borges, J.T., Queiroz, M.N. y Barbosa, M. 2009a. Parasitic infections in pirarucu fry, Arapaima gigas Schinz, 1822 (Arapaimatidae) kept in a semi-intensive fish farm in Central Amazon, Brazil. Veterinarski Arhiv 79(5): 499-507.

Araújo, C.D., Tavares-Dias, M., Gomes, A.L.S., Andrade, S.M.S., Lemos, J.R.G., Oliveira, A.D. y Affonso, E.G. 2009b. Infecções parasitárias e parâmetros sanguíneos em Arapaima gigas Schinz, 1822 (Arapaimidae) cultivados no estado do Amazonas, Brasil. En: Tavares-Dias, M. Editor. Manejo e sanidade de peixes em cultivo. Macapá, Amapá. Avnimelech, Y. 2009. Biofloc technology. A practical guide book. The World Aquaculture Society, Baton Rouge. 
Bowker, J.D., Carty, D. y Dotson, M.M. 2012. Efficacy of $35 \%$ PEROX-AID (hydrogen peroxide) in reducing an infestation of Gyrodactylus salmonis in freshwater-reared rainbow trout. North American Journal of Aquaculture 74(2): 154-159.

Boyd, C.E. y Massaut, L. 1999. Risks associated with the use of chemicals in pond aquaculture. Aquacultural Engineering 20(2): 113-132.

Crab, R., Defoirdt, T., Bossier, P. y Verstraete, W. 2012. Biofloc technology in aquaculture: beneficial effects and future challenges. Aquaculture 356: 351-356.

Da Costa, J.C., Valladão, G.M.R., Pala, G., Gallani, S.U., Kotzent, S., Crotti, A.E.M., Fracarolli, L., da Silva, J.J.M. y Pilarski, F. 2017. Copaifera duckei oleoresin as a novel alternative for treatment of monogenean infections in pacu Piaractus mesopotamicus. Aquaculture 471: 72-79.

Food and Agriculture Organization of the United Nations (FAO). 2016. El estado mundial de la pesca y la acuicultura 2016. Contribución a la seguridad alimentaria y la nutrición para todos, Roma.

Balbuena, E.D. 2011. Manual básico de piscicultura. Editorial Food and Agriculture Organization of the United Nations (FAO), Asunción.

Forwood, J.M., Harris, J.O. y Deveney, M.R. 2013. Efficacy of current and alternative bath treatments for Lepidotrema bidyana infecting silver perch, Bidyanus bidyanus. Aquaculture 416: 65-71.

Franceschini, L., Zago, A.C., Schalch, S.H.C., Garcia, F., Romera, D.M. y Silva, R.J.D. 2013. Parasitic infections of Piaractus mesopotamicus and hybrid (P. mesopotamicus $\mathrm{x}$ Piaractus brachypomus) cultured in Brazil. Revista Brasileira de Parasitologia Veterinária 22(3): 407-414.

Hirazawa, N., Tsubone, S. y Takano, R. 2016. Anthelmintic effects of 75ppm hydrogen peroxide treatment on the monogeneans Benedenia seriolae, Neobenedenia girellae, and Zeuxapta japonica infecting the skin and gills of greater amberjack Seriola dumerili. Aquaculture 450: 244-249.

Huang, A.G., Yi, Y.L., Ring, F., Lu, L., Zhang, Q.Z. y Wang, G. X. 2013. Screening of plant extracts for anthelmintic activity against Dactylogyrus intermedius (Monogenea) in goldfish (Carassius auratus). Parasitology Research 112(12): 4065-4072.
Kasai, H., Yoshimizu, M. y Ezura, Y. 2002. Disinfection of water for aquaculture. Fisheries Science 68(sup1): 821-824.

Kasai, H., Ishikawa, A., Hori, Y., Watanabe, K.I. y Yoshimizu, M. 2000. Disinfectant effects of electrolyzed salt water on fish pathogenic bacteria and viruses. Nippon Suisan Gakkaishi 66(6): 1020-1025.

Mendoza-Franco, E.F., Scholz, T. y Rozkošná, P. 2010. Tucunarella $\mathrm{n}$. gen. and other dactylogyrids (Monogenoidea) from cichlid fish (Perciformes) from Peruvian Amazonia. Journal of Parasitology 96(3): 491-498.

Monroy-Dosta, M.D.C., Lara-Andrade, D., Castro-Mejía, J., Castro-Mejía, G. y Coelho-Emerenciano, M.G. 2013. Composición y abundancia de comunidades microbianas asociadas al biofloc en un cultivo de tilapia. Revista de Biología Marina y Oceanografía 48(3): 511-520.

Pahor-Filho, E., Miranda-Filho, K.C. y Júnior, J.P. 2012. Parasitology of juvenile mullet (Mugil liza) and effect of formaldehyde on parasites and host. Aquaculture 354: 111-116.

Raja, R.A. y Jithendran, K.P. 2015. Aquaculture Disease Diagnosis and Health Management. En: Perumal, S., Thirunavukkarasu, A.R. y Pachiappan, P. Editor. Advances in Marine and Brackishwater Aquaculture. Springer, Nueva Delhi.

Sarmiento, J. y Rodríguez, A. 2013. Lerneosis en alevinos de Prochilodus magdalenae, Prochilodontidae, cultivados en laboratorio. Intropica 8: 99-103.

Sitjà-Bobadilla, A., de Felipe, M. C. y Alvarez-Pellitero, P. 2006. In vivo and in vitro treatments against Sparicotyle chrysophrii (Monogenea: Microcotylidae) parasitizing the gills of gilthead sea bream (Sparus aurata L.). Aquaculture 261(3): 856-864.

Takemoto, R.M., Lizama, M.A., Guidelli, G.M. y Pavanelli, G.C. 2004. Parasitos de peixes de águas continentais. En: Ranzani-Paiva, M., Takemoto, R. Lizama, M. Editores. Sanidade de organismos aquáticos. Editora Varela, São Paulo.

Tavares-Dias, M., Moraes, F.R. y Martins, M.L. 2008. Hematological assessment in four Brazilian teleost fish with parasitic infections, collected in feefishing from Franca, São Paulo, Brazil. Boletim do Instituto de Pesca 34(2): 189-196. 
Thoney, D.A. y Hargis, W.J. 1991. Monogenea (Platyhelminthes) as hazards for fish in confinement. Annual Review of Fish Diseases 1: 133-153.

Umeda, N., Nibe, H., Hara, T. y Hirazawa, N. 2006. Effects of various treatments on hatching of eggs and viability of oncomiracidia of the monogenean Pseudodactylogyrus anguillae and Pseudodactylogyrus Bini Aquaculture 253(1): 148-153.

Valladão, G.M.R., Gallani, S.U. y Pilarski, F. 2016. South American fish for continental aquaculture. Reviews in Aquaculture DOI: 10.1111/raq.12164.
Valladão, G.M.R., Gallani, S.U. y Pilarski, F. 2015. Phytotherapy as an alternative for treating fish disease. Journal of Veterinary Pharmacology and Therapeutics 38(5): 417-428.

Wilfart, A., Prudhomme, J., Blancheton, J.P. y Aubin, J. 2013. LCA and emergy accounting of aquaculture systems: Towards ecological intensification. Journal of Environmental Management 121: 96-109.

Fecha de recepción: 08/07/2017

Fecha de aceptación: 20/03/2018

Publicado en línea: 28/05/2018

Para citar este artículo: Cala Delgado, D.L., Álvarez Rubio, N.C., Muñoz Rodríguez, F.A. Blanco Torres, C.A. y Yunis Aguinaga, Y. 2018. Diagnóstico clínico de monogeneos en alevinos de piscicultura intensiva en Arauca. Intropica 13(1): 